\title{
FPGA Realization of Spherical Chaotic System with Application in Image Transmission
}

\author{
Jose Cruz Nuñez-Perez $\left(\mathbb{D},{ }^{1}\right.$ Vincent Ademola Adeyemi ${ }^{(D)},{ }^{1}$ Yuma Sandoval-Ibarra $\mathbb{D}^{2}{ }^{2}$ \\ F. Javier Pérez-Pinal $\mathbb{D}^{3},{ }^{3}$ and Esteban Tlelo-Cuautle $\mathbb{D}^{4}$
}

\author{
${ }^{1}$ Instituto Politécnico Nacional, IPN-CITEDI, Tijuana, Baja California 22435, Mexico \\ ${ }^{2}$ Universidad Politécnica De Lázaro Cárdenas, UPLC, Lázaro Cárdenas, Michoacán 60950, Mexico \\ ${ }^{3}$ Tecnológico Nacional De México, Instituto Tecnológico De Celaya, Celaya, Guanajuato 38010, Mexico \\ ${ }^{4}$ Instituto Nacional De Astrofísica, Óptica y Electrónica, INAOE, San Andrés Cholula, Puebla 72840, Mexico
}

Correspondence should be addressed to Jose Cruz Nuñez-Perez; nunez@citedi.mx

Received 12 February 2021; Revised 12 March 2021; Accepted 1 April 2021; Published 14 April 2021

Academic Editor: Sanbo Ding

Copyright ( 92021 Jose Cruz Nuñez-Perez et al. This is an open access article distributed under the Creative Commons Attribution License, which permits unrestricted use, distribution, and reproduction in any medium, provided the original work is properly cited.

\begin{abstract}
This paper considers a three-dimensional nonlinear dynamical system capable of generating spherical attractors. The main activity is the realization of a spherical chaotic attractor on Intel and Xilinx FPGA boards, with a focus on implementation of a secure communication system. The first major contribution is the successful synchronization of two chaotic spherical systems, in VHDL program, in a master-slave topology using Hamiltonian forms. The synchronization errors show that the two spherical chaotic systems synchronize in a very short time after which the error signals become zero. The second major contribution is the FPGA realization of a spherical chaotic attractor-based secure communication system, which involves encrypting both grayscale and RGB images with chaos and diffusion key at the transmitting system, sending the encrypted image via the state variables, and reconstructing the encrypted image at the receiving system. The Intel Stratix III and Xilinx Artix-7 AC701 results are the same as those of MATLAB. The statistical analyses of the encrypted and received images show that the implemented system is very effective, as it reveals high degree of randomness in the encrypted images with the entropy test, and the obtained correlation coefficient, which is zero, removes relativity between the original and encrypted images. Finally, the transmission system fully recovers the original grayscale and RGB images without loss of information.
\end{abstract}

\section{Introduction}

In the past few decades, dynamical systems based on chaos theory have begun to be implemented in various fields, such as computer science, economics, robotics, physics, medicine, biomedical, and so on, due to their potential applications. This has prompted researchers into the study of nonlinear dynamical systems [1-3]. Typically, a nonlinear system is regarded as a system in which the laws governing the time evolution of its state variables depend on the values of these variables in a manner that deviates from proportionality [4]. Some of the earliest researchers of nonlinear systems are Henri Poincaré, who studied nonlinear dynamics as $n$-body problem in celestial mechanics, Aleksandr Mikhailovich
Lyapunov, who studied the stability of nonlinear dynamical systems, and Balthasar van der Pol, who studied limit cycles in relation with oscillator dynamics. Others are Georg Duffing, who modelled a dynamic chaotic system using second-order nonlinear differential equations, Hendrik Wade Bode, who studied the asymptotic representation of the frequency response, and Edward Norton Lorenz, who gave us the chaos theory and the famous butterfly effect [5].

Nonlinear systems can exhibit limit cycle, fractal, and irregular chaotic behaviors. Others are bifurcation, multistability, and nonlinear divergent behaviors [6, 7]. A limit cycle is when the nonlinear system has a nontrivial periodic output; fractal is when there is a self-similar geometric pattern exhibited on the phase plane, and this self-similar 
geometric pattern is repeated at ever smaller scales to produce irregular shapes and surfaces that cannot be represented by classical geometry; and irregular chaotic behavior is when the nonlinear system is sensitive to its initial condition; a state orbit is dense and consists of dense periodic orbits, but fractal patterns do not exhibit on the phase plane. The qualitative change in the dynamics of the nonlinear system as parameters vary is called bifurcation; the presence of two or more stable states is called multistability, while the nonlinear divergent behavior occurs when some state variables tend to infinity, but the corresponding linear part is strictly stable.

Most real physical systems and phenomena are inherently nonlinear in nature. For example, gravitational and electrostatic attraction, elasticity, bio-mechanics, fluid and plasma mechanics, gas dynamics, chemical reactions, robot, aircraft and spacecraft control, combustion, and biological systems are all governed by nonlinear models. Hence, the nonlinear system is an important subject of interest to engineers, physicists, mathematicians, and scientists. Some engineering and scientific applications of the nonlinear systems can be found in [8-17]. The mathematical models of the nonlinear systems are represented by nonlinear differential equations. One of the challenges facing the nonlinear systems is the generation of the solutions and their characterization. The evaluation of the behavior of nonlinear systems is important, and it shows the actual nature of the systems. But due to the nonlinearity and complexity of the nonlinear systems, it is very difficult to derive the analytical or closed-loop solutions for the systems. Therefore, in solving or simulating the nonlinear systems, researchers rely on approximate or numerical methods, which provide approximate results for the systems $[4,18]$.

The irregular chaotic behavior of the nonlinear systems is one property that has been widely exploited, giving rise to modelling of several nonlinear dynamical systems. As the system dimension for chaos ranges from 1, 2 to multidimensions, several simple and complex chaotic systems have been constructed and more are being developed. For example, Lorenz proposed a 3-D system [19] in 1963, which is recognized as the first chaotic model. The Chen oscillator [20] was proposed as a special case of a generalized Lorenz canonical form. The Rössler systems [21] were proposed as prototype equations with the minimum ingredients for continuous-time chaos. The Rössler systems are well-known for their simplicity because they have single quadratic term. In addition, Rössler also proposed four-dimensional systems [22]. It is hereby noted that new chaotic systems can now be constructed by systematic examination of generic 3-D quadratic autonomous ordinary differential equations through exhaustive computer search [23], analytic method involving chaotification of a nonchaotic system via feedback anti-control developed by Chen [24], Shilnikov analytic criterion [25], or any other proven techniques. Constructed chaotic systems are usually analyzed for chaotic dynamics by phase diagrams, Lyapunov exponents, bifurcation diagrams, fractal dimension, time series, information entropy, and so on $[26,27]$.
Due to the potential in chaos, a notable area that has attracted so many interests from researchers is chaos-based communication systems, which is based on synchronization of chaotic oscillators as a backbone of the communication platform [28-31]. The synchronization of chaos occurs when a chaotic system (master system) drives another identical or nonidentical chaotic system (slave system), such that the output of the slave system follows the output of the master system, asymptotically. The synchronization between the chaotic master system and the chaotic slave system can be achieved with different methods, such as Pecora and Carroll, Ott-Grebogi-Yorke (OGY), Hamiltonian forms and observer approach, open-plus-close-loop, and so on. Once synchronization is achieved, transmission of information is possible as seen for the first time in [32].

Theoretical and numerical implementations of new chaotic oscillators continue to be a subject of interest, and the significance of finding new chaotic oscillators cannot be overemphasized. First, it is important to find new chaotic systems that have high positive Lyapunov exponent, which is beneficial to developing secure physical systems such as communication systems. Second, it is also important to have new chaotic oscillators that possess multistable states, which allow easy control of chaos in various application fields such as laser physics and optics, electrical engineering, and telecommunications. Third, it is beneficial to have several options to choose from while developing real physical systems with chaotic oscillators. Furthermore, researchers have also moved on, over the years, to experimental verifications of chaotic oscillators via electronic implementations for the purpose of creating real-world chaos-based applications. The options for circuit implementation vary with different kinds of potential electronic building blocks. For instance, analog implementation with operational amplifiers (OPAMPs) was a popular choice in most of the earlier circuit implementations, as seen in [33, 34] for multiscroll chaotic oscillators and in $[35,36]$ for hyperchaotic oscillators. However, the OPAMP-based implementation has some drawbacks. For example, it is difficult to realize nonlinear resistors and capacitors appropriate for hardware implementation of chaotic attractors [34]. Also, OPAMPs have low slew rate [37]. These and other obstacles prompted the need for better electronic implementation.

The field-programmable gate arrays (FPGAs) have become the most attractive and popular choice for electronic realization of chaotic systems, owing to some advantages inherent in them. For example, FGPAs offer better performance as they can perform parallel processing at a faster rate. FPGAs are reprogrammable, even after the circuit has been designed and implemented. This important characteristic not only makes FPGAs reusable but also makes them adaptable, extremely cost effective, and perfect choice for prototyping purposes. Furthermore, the design process of the FPGAs, performed by hardware description languages (HDLs), is extremely fast and efficient [38].

There are many investigations that have been carried out on FPGA realization of chaotic systems and its applications. For example, in [39], a high-speed FPGA-based Lü-Chen chaotic oscillator was designed in VHDL using Heun 
numerical method and realized on Xilinx Virtex- 6 chip. The results of the FPGA-based implementation were found to corroborate the computer-based results from MATLAB. The authors in [40] designed a 3-D chaotic system, applying the Euler method for its discrete-time generation, and implemented a secure color image encryption system based on the chaotic signals. These were realized on an Altera FPGA DE2115 board. A novel artificial neural network-based chaotic true random number generator (TRNG) was realized in a Xilinx Virtex-6 in [41]. The numerical solution of the underlying Pehlivan-Uyaroglu chaotic system was obtained using the fifth-order Runge-Kutta algorithm. Furthermore, the investigation in [42] includes the implementation of a new one-parameter chaotic system on Altera Cyclone IV FPGA board. The applied numerical method for solving the dynamical system in VHDL was the forward Euler method. The work was extended to create a secure communication system with the chaotic system and realize it on the FPGA board. A coupled map lattice (CML), which can exhibit extremely complex spatiotemporal chaos, was applied to create a multimedia cryptosystem in [43]. To improve the encryption speed, the cryptosystem was realized in a Xilinx Spartan-3 device. Other related work on FPGA realization of nonlinear dynamical systems and their application in secure communication systems can be found in [44-50].

In this work, the authors investigate a 3-D nonlinear system proposed in [51], which generates spherical chaotic attractor. The goals of this investigation are to emulate for the first time, to the best of the authors' knowledge [52], on FPGA, the spherical chaotic system and also implement a chaos-based information transmission system. By doing these, the following contributions to the state of the art are hereby emphasized:

(1) FPGA realization of the 3-D spherical chaotic system in a design process performed on Intel's Stratix III and Xilinx Artix-7 AC701, using VHDL with a word length of 32-bit. The VHDL implementations on the FPGA boards completely agree with the simulation done in MATLAB.

(2) Successful synchronization and FPGA realization of two spherical chaotic systems in a master-slave configuration, using VHDL with a word length of 32bit for the hardware design on Intel's Stratix III and Xilinx Artix-7 AC701. The slave system serves as the observer of states whose state variables approximate the master system. Both the VHDL and MATLAB synchronization results are in complete agreement.

(3) The FPGA realization of a spherical chaotic attractorbased secure communication system to successfully encrypt and transmit grayscale and RGB images, using VHDL with a word length of 32-bit for the hardware design on Intel's Stratix III and Xilinx Artix7 AC701. The encrypted images have no relativity with the original images, and the original images were fully recovered without loss of information. The VHDL implementations on the FPGA boards are in consonance with the MATLAB simulation.
The paper is organized as follows. Section 2 contains the theoretical framework, showcasing the 3-D nonlinear chaotic system that generates spherical attractors. Section 3 shows the Hamiltonian form method for synchronizing the spherical systems in a master-slave topology. In Section 4, the results of the FPGA realization of the spherical chaotic attractor, master-slave synchronization, and image encryption and transmission are presented. The discussion of the results obtained is found in Section 5. Lastly, Section 6 contains the conclusions.

\section{Spherical Chaotic System}

The authors in [51] proposed a smooth quadratic autonomous chaotic system from an earlier system constructed in [53] based on the Shilnikov criterion [54, 55], which is an analytic method for proving chaos in nonlinear dynamical systems [25]. The Shilnikov criterion was proposed in 1965 by a Russian Mathematician, Leonid Pavlovich Shilnikov. L.P. Shilnikov was an originator of the theory of global bifurcations of multidimensional dynamical systems and also one of the founders of the mathematical theory of dynamical chaos and strange attractors. The two theorems in the Shilnikov criterion provide a theoretical foundation for classification of chaos. One theorem is based on the presence of heteroclinic orbit while the other is based on the existence of homoclinic orbit. Let us consider a third-order autonomous system given in the following equation:

$$
\frac{d x}{d t}=f(x), \quad x \in \mathbf{R}^{3}, \quad t \in \mathbf{R},
$$

where the vector field $f(x): \boldsymbol{R}^{3} \longrightarrow \boldsymbol{R}^{3}$ is r-times differentiable $(k \geq 1)$ with a continuous derivative $C^{k}$. Let $x_{\mathrm{e}} \in \boldsymbol{R}^{\mathbf{3}}$ be an equilibrium point of equation (1); then, $x_{e}$ is called a saddle focus if the eigenvalues of the Jacobian $J$ of $f(x)$ evaluated at $x_{e}$ are of the form

$$
\lambda_{1}=\alpha, \lambda_{2,3}=\beta \pm i \gamma, \quad \alpha \beta<0, \quad \gamma \neq 0,
$$

where $\alpha, \beta$, and $\gamma$ are real.

Theorem 1 (Shilnikov homoclinic theorem). For a 3-D autonomous system in equation (1), let $x_{e}$ be a saddle focus equilibrium point whose eigenvalues satisfy $\alpha>|\beta|>0$, and there exists a homoclinic orbit connected at $x_{e}$. Then, the 3-D autonomous system has horseshoe chaos [54, 55].

Theorem 2 (Shilnikov heteroclinic theorem). Suppose that two distinct equilibrium points $x_{e}^{1}$ and $x_{e}^{2}$ of a 3-D autonomous system in equation (1) are saddle foci, whose eigenvalues at these points are $\alpha_{n}$ and $\beta_{n} \pm i \gamma_{n}(n=1,2)$, which satisfy the Shilnikov inequality $\alpha_{n}>\beta_{n}>0, n=1,2$, under the constraint $\beta_{1} \beta_{2}>0$ or $\alpha_{1} \alpha_{2}>0$. Suppose also that there exists a heteroclinic orbit connecting points $x_{e}^{1}$ and $x_{e}^{2}$. Then, the 3-D autonomous system has horseshoe chaos [54, 55].

The spherical chaotic system, proposed in [51] and used in this work, is perturbed by a hyperbolic tangent function as given in the following equation: 


$$
\left.\begin{array}{l}
\dot{x}=a_{1} x-a_{2} y+a_{3} z+2\left(\frac{1-e^{-200 \sin y}}{1+e^{-200 \sin y}}\right) \\
\dot{y}=-d x z+b+e x \\
\dot{z}=c_{1} x y+c_{2} y z+c_{3} z+c
\end{array}\right\},
$$

where $a_{i}, c_{i}(1 \leq i \leq 3), d, b, c$, and $e$ are all real parameters, while $x, y$, and $z$ are the state variables.

In this work, unless otherwise stated, the values of the system parameters are $a_{1}=-4.1, a_{2}=1.2, a_{3}=13.45$, $b=0.161, c=3.5031, c_{1}=2.76, c_{2}=0.6, c_{3}=13.13, d=1.6$, and $e=0$, while the initial condition, randomly chosen in the basin of attraction of the system, is $x_{0}=(-0.04,-15.8,-1.4)$. With the parameter values and the initial condition, the chaotic system in equation (3) has the following Lyapunov exponents: $L_{1}=0.0207, L_{2}=0$, and $L_{3}=-0.0693$. The phase space portraits of the chaotic system are presented in Figure 1 in both 2-D and 3-D, respectively. The fourth-order Runge-Kutta method was applied in this work to perform the numerical integration of the system.

\section{Hamiltonian Forms}

The Hamiltonian forms are applied to achieve the synchronization of two spherical attractors of the chaotic oscillator given in equation (3) in a master-slave topology [56]. This synchronization method is chosen because it allows systematic synchronization and simplifies the problem of deciding the nature of the output signal to transmit. Also, it does not require the use of the Lyapunov exponents. Moreover, it does not require that the initial conditions belong to the same basin of attraction. These are some of the advantages that give Hamiltonian forms an edge over other synchronization methods. Therefore, the method can be easily applied to synchronize several chaotic oscillators. The slave system serves as the observer of states whose state variables will approximate the master system.

Mathematically, the master and slave of a dynamical system are expressed in Hamiltonian form as follows:

$$
\dot{x}=f(x),
$$

where $\dot{x} \in \mathbf{R}^{\mathbf{n}}$ is the state variable and $f: \mathbf{R}^{\mathbf{n}} \longrightarrow \mathbf{R}^{\mathbf{n}}$ is the nonlinear function. The dynamical system in equation (4) can be written also as

$$
\dot{x}=A \frac{\partial H}{\partial x}+\mathscr{F}(x),
$$

where $A=\left(\left(A-A^{T} / 2\right)+\left(\left(A+A^{T}\right) / 2\right)\right)$. Therefore,

$$
\dot{x}=\frac{A-A^{T}}{2} \frac{\partial H}{\partial x}+\frac{A+A^{T}}{2} \frac{\partial H}{\partial x}+\mathscr{F}(x) .
$$

Let $\mathscr{J}(x)=\left(A-A^{T} / 2\right)$ and $\mathcal{S}(x)=\left(A+A^{T} / 2\right)$; then, equation (6) can be written in the generalized Hamiltonian canonical form as

$$
\dot{x}=\mathscr{J}(x) \frac{\partial H}{\partial x}+\mathcal{S}(x) \frac{\partial H}{\partial x}+\mathscr{F}(x), \quad x \in \mathbf{R}^{\mathbf{n}},
$$

where $H(x)=(1 / 2) x^{T} \mathscr{M} x$ denotes a positive smooth energy function definite in $\mathbf{R}^{\mathbf{n}}, \mathscr{M}$ is a constant, symmetric positive definite matrix, and hence, $\partial H / \partial x=\mathscr{M} x . \partial H / \partial x$ is the column gradient vector of $H(x)$, while matrix $\mathscr{J}(\mathrm{x})$ satisfies $\mathscr{J}(x)+\mathscr{J}^{T}(x)=0$ and $\mathcal{S}(x)$ satisfies $\mathcal{S}(x)=$ $\mathcal{S}^{T}(x)$ for all $x \in \mathbf{R}^{\mathbf{n}}$. The vector field $\mathscr{J}(x)(\partial H / \partial x)$ exhibits the conservative part and $\delta(x)$ represents the nonconservative part of the system.

The master system in the case of the observer design approach, which is a special class of the generalized Hamiltonian forms with destabilizing vector field and output $y(t)$, is given by the following equation:

$$
\left\{\begin{array}{l}
\dot{x}=\mathscr{J}(y) \frac{\partial H}{\partial x}+\mathcal{S}(y) \frac{\partial H}{\partial x}+\mathscr{F}(y), \quad x \in R^{n} \\
y=\mathscr{C} \frac{\partial H}{\partial x}, \quad y \in R^{m}
\end{array}\right.
$$

where $\mathcal{S}$ is a constant symmetric matrix and $\mathscr{C}$ is a constant matrix.

On the other hand, the slave system, which is the dynamical nonlinear state observer, is defined as follows. The estimate of the state vector $x$ is denoted by $\dot{\xi}$, and the estimated output is denoted by $\eta$. Therefore, the slave system is

$$
\left\{\begin{array}{l}
\dot{\xi}=\mathscr{J}(y) \frac{\partial H}{\partial \xi}+\mathcal{S}(y) \frac{\partial H}{\partial \xi}+\mathscr{F}(y)+K e_{y}, \quad \xi \in \mathbf{R}^{\mathbf{n}} \\
\eta=\mathscr{C} \frac{\partial H}{\partial \xi}, \quad \eta \in \mathbf{R}^{\mathbf{m}}
\end{array}\right.
$$

where $\mathscr{K}$ is the gain of the observer and $e_{y}=y-\eta$ is the output estimation error. The state estimation error is defined by $e=x-\xi$. Based on the foregoing mathematical analysis, the master and slave systems of spherical chaotic system (3) are constructed as follows:

$$
\begin{aligned}
& \mathscr{J}(x)=\left[\begin{array}{ccc}
0 & \frac{-a_{2}-e}{2} & \frac{a_{3}}{2} \\
\frac{a_{2}+e}{2} & 0 & \frac{-c_{1} x-d x}{2} \\
-\frac{a_{3}}{2} & \frac{c_{1} x+d x}{2} & 0
\end{array}\right], \\
& \mathcal{S}(x)=\left[\begin{array}{ccc}
\frac{a_{1}}{2} & \frac{-a_{2}+e}{2} & \frac{a_{3}}{2} \\
\frac{a_{2}+e}{2} x-d x \\
\frac{a_{3}}{2} & \frac{c_{1} x-d x}{2} & c_{2} y+c_{3}
\end{array}\right], \\
& \mathscr{F}(x)=\left[\begin{array}{cc}
\left.\frac{1-e^{-200 \sin y}}{1+e^{-200 \sin y}}\right) \\
b
\end{array}\right] .
\end{aligned}
$$




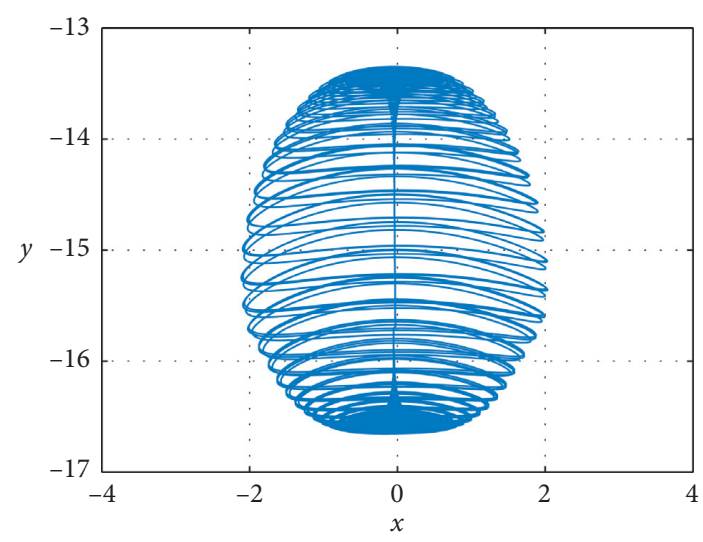

(a)

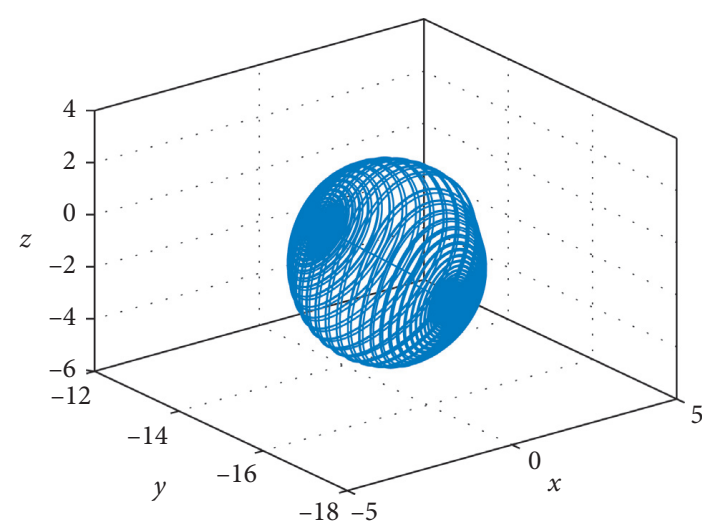

(b)

FIGURE 1: Spherical attractors in 2-D and 3-D of the chaotic system given in equation (3).

From equation (8),

$$
\left[\begin{array}{c}
\dot{x} \\
\dot{y} \\
\dot{z}
\end{array}\right]=\left[\begin{array}{ccc}
0 & \frac{-a_{2}-e}{2} & \frac{a_{3}}{2} \\
\frac{a_{2}+e}{2} & 0 & \frac{-c_{1} x-d x}{2} \\
-\frac{a_{3}}{2} & \frac{c_{1} x+d x}{2} & 0
\end{array}\right] \frac{\partial H}{\partial x}+\left[\begin{array}{ccc}
a_{1} & \frac{-a_{2}+e}{2} & \frac{a_{3}}{2} \\
\frac{-a_{2}+e}{2} & 0 & \frac{c_{1} x-d x}{2} \\
\frac{a_{3}}{2} & \frac{c_{1} x-d x}{2} & c_{2} y+c_{3}
\end{array}\right] \frac{\partial H}{\partial x}+\left[\begin{array}{c}
2\left(\frac{1-e^{-200 \sin y}}{1+e^{-200 \sin y}}\right) \\
b \\
c
\end{array}\right] \text {, }
$$

where $H(x)=(1 / 2)\left[x^{2}+y^{2}+z^{2}\right]$ and the gradient vector is $\partial H / \partial x=\left[\begin{array}{l}x \\ y \\ z\end{array}\right]$.

Therefore, the master system is

$$
\left[\begin{array}{c}
\dot{x} \\
\dot{y} \\
\dot{z}
\end{array}\right]=\left[\begin{array}{c}
a_{1} x-a_{2} y+a_{3} z \\
-d x z+e x \\
-c_{1} x y+c_{2} y z+c_{3} z
\end{array}\right]+\left[\begin{array}{c}
2\left(\frac{1-e^{-200 \sin y}}{1+e^{-200 \sin y}}\right) \\
b \\
c
\end{array}\right] .
$$

Also, according to equation (9), the slave system is represented by

$$
\left[\begin{array}{l}
\dot{\xi}_{1} \\
\dot{\xi}_{2} \\
\dot{\xi}_{3}
\end{array}\right]=\left[\begin{array}{ccc}
0 & \frac{-a_{2}-e}{2} & \frac{a_{3}}{2} \\
\frac{a_{2}+e}{2} & 0 & \frac{-c_{1} x-d x}{2} \\
-\frac{a_{3}}{2} & \frac{c_{1} x+d x}{2} & 0
\end{array}\right] \frac{\partial H}{\partial \xi}+\left[\begin{array}{ccc}
a_{1} & \frac{-a_{2}+e}{2} & \frac{a_{3}}{2} \\
\frac{-a_{2}+e}{2} & 0 & \frac{c_{1} x-d x}{2} \\
\frac{a_{3}}{2} & \frac{c_{1} x-d x}{2} & c_{2} y+c_{3}
\end{array}\right] \frac{\partial H}{\partial \xi}+\left[\begin{array}{c}
2\left(\frac{1-e^{-200 \sin y}}{1+e^{-200 \sin y}}\right) \\
b \\
c
\end{array}\right]+\left[\begin{array}{c}
k_{1} \\
k_{2} \\
k_{3}
\end{array}\right] e_{y},
$$


which, by simplification, becomes

$$
\left[\begin{array}{c}
\dot{y} 1 \\
\dot{y} 2 \\
\dot{y 3}
\end{array}\right]=\left[\begin{array}{c}
a_{1} x-a_{2} y+a_{3} z \\
-d x z+e x \\
-c_{1} x y+c_{2} y z+c_{3} z
\end{array}\right]+\left[\begin{array}{c}
2\left(\frac{1-e^{-200 \sin y}}{1+e^{-200 \sin y}}\right) \\
b \\
c
\end{array}\right]+\left[\begin{array}{c}
k_{1} \\
k_{2} \\
k_{3}
\end{array}\right] e_{y}
$$

The synchronization is considered successful according to the following definition and theorems, created in [57] from the perspective of passivity-based state observer design in the context of generalized Hamiltonian systems including dissipative and destabilizing vector fields.

Definition 1. The basic condition for the slave to synchronize with the master in Hamiltonian form is when

$$
\lim _{t \longrightarrow \infty}\|x(t)-\xi(t)\|=0,
$$

regardless of what the initial conditions $x(0)$ and $\xi(0)$ are [57].

Theorem 3. The state $x$ of the nonlinear system in equation (8) can be globally, exponentially, and asymptotically estimated by the state $\xi$ of the nonlinear observer in equation (9) if the pair of matrices $(\mathscr{C}, \mathcal{S})$ is observable [57].

Theorem 4. The state $x$ of the nonlinear system in equation (8) can be globally, exponentially, and asymptotically estimated by the state $\xi$ of the nonlinear observer in equation (9) if and only if there exists a constant matrix $\mathscr{K}$ such that the symmetric matrix

$$
\begin{aligned}
{[W-K C]+[W-K T]^{T} } & =[S-K C]+[S-K C]^{T} \\
& =2\left[S-\frac{1}{2}\left(K C+C^{T} K^{T}\right)\right],
\end{aligned}
$$

is negative definite [57].

\section{Results}

This section contains the results obtained in this investigation in the master-slave synchronization and image encryption and transmission, including the FPGA device resources consumed.

In this investigation, all the activities were first modelled in VHDL using the Quartus II/ModelSim design software version 13.1 and then emulated on FPGA device. A 32-bit word was used in the VHDL programming in order to maintain fidelity to the original implementation of this work in MATLAB version 2016b. That is, 1 bit for the sign, 6 for the integer, and 25 for the fractional part. Moreover, to avoid reducing the diameter of the oscillator, the implementation of the hyperbolic tangent function in the system in equation (3) in VHDL was done using a lookup table (LUT), instead of calculating it by means of LUTs for the functions $\sin (y)$ and $e^{y}$. This option results in a single LUT of 512 samples of 8 bits each. The results of the VHDL implementation, simulated in ModelSim and compared with MATLAB, are presented in this section.

4.1. Master-Slave Synchronization. The master-slave synchronization is illustrated in Figure 2, which shows the coupling between the master system in equation (12) and slave system in equation (13).

The multiplexer MUX handles the setting of the initial conditions for both the master and slave systems and allows performing the needed iterations of the equations representing the master and slave systems using the fourth-order Runge-Kutta method. The pin Sel controls the number of iterations to perform, while the registers store the outputs of the master and slave systems, and the subtractors compute the synchronization errors $e_{1}, e_{2}$, and $e_{3}$. Following Theorem 4 , the gains of the observer used in the synchronization were $k_{1}=2, k_{2}=7$, and $k_{3}=5$. The synchronization is simulated in MATLAB and compared with the VHDL-generated results in ModelSim. Both simulations have the same initial conditions $x_{0}=(-0.04,-15.8,-1.4)$ for the master system and $y_{0}=(-0.12,-12.41,-2.1)$ for the slave system and a total of $35 \times 10^{4}$ samples each. The initial conditions were randomly chosen in the basin of attraction of the chaotic system, out of the many possible values. The master and slave chaotic systems are sensitive to the chosen initial conditions. This was confirmed by computing the Lyapunov exponents, which in each case has one positive value.

In Figure 3 are found the waveforms of the master system (blue) and slave system (magenta) in both MATLAB and ModelSim. The VHDL-generated synchronization errors are presented in Figure 4(b) while the MATLAB implementation is shown in Figure 4(a). The errors $e_{1}=x_{1}-\xi_{1}$ (red), $e_{2}=x_{2}-\xi_{2}$ (blue), and $e_{3}=x_{3}-\xi_{3}$ (magenta), in which $x_{i}$ represents the master while $\xi_{i}$ denotes the slave.

The results of the Hamiltonian forms synchronization in Figures 3 and 4 show that the master system, slave system, and the synchronization errors obtained from MATLAB are the same as the output from VHDL/ModelSim. Specifically, as it is seen in Figure 4, the master and slave signals were synchronized in a very short time after which the error signals $e_{1}, e_{2}$, and $e_{3}$ became zero. In other words, the master and slave systems became synchronized as soon as the 


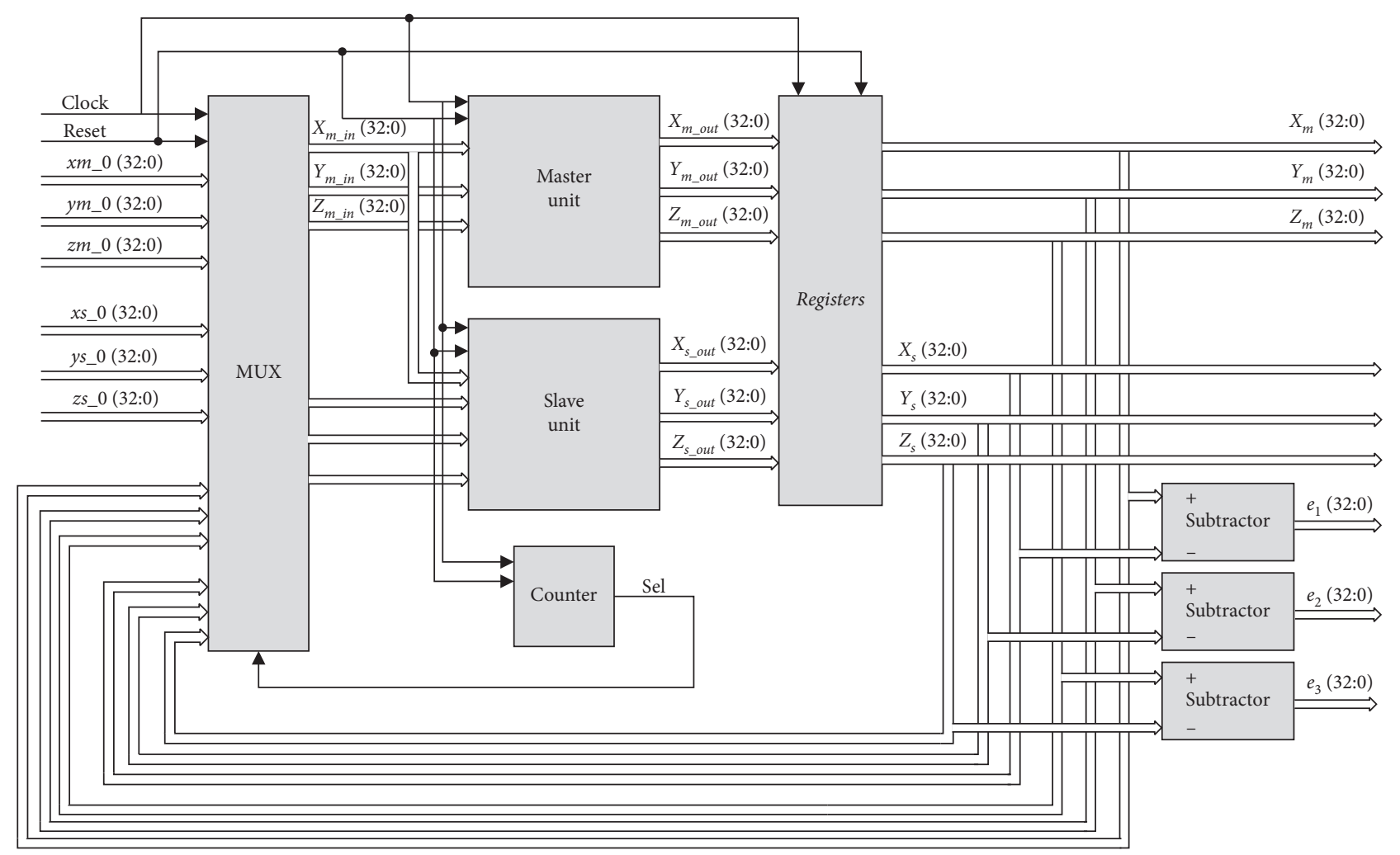

FIgURE 2: Synchronized spherical attractors in master-slave topology.

simulation started, around 1,100th iteration, just $0.3 \%$ of the total iterations. Therefore, it could be concluded that the ratio of the master and the slave states $x_{i}$ and $\xi_{1}$ is 1 after a very short time.

4.2. Image Encryption and Transmission. Once the master and slave systems are synchronized, the implementation of a secure communication system for image transmission is achievable. In the present investigation, the images transmitted were grayscale of size $640 \times 480$ pixels and RGB of size $320 \times 240$ pixels. The MATLAB-Simulink block diagram of the secure communication system is described in Figure 5.

The methodology involved in the image encryption and transmission begins in the master system, which is the transmitter, whereby the original image undergoes a robust encryption with the chaotic master signal $X_{m}$ and diffusion key using the $\mathrm{X}-\mathrm{OR}$ operator. The transmitter generates a secure, encrypted image $I M_{t}$ and transmits same to the slave system, which is the receiver, to begin the image reconstruction process. The decryption methodology at the receiver is the inverse of the encryption procedure. Because the slave system approximates the master system, the original image is fully reconstructed and recovered in $\mathrm{IM}_{r}$.

For the best results according to the correlation between the original and encrypted images (see Table 1), the obtained waveforms in MATLAB and ModelSim from the original (red), encrypted (blue), and recovered (magenta) images are shown in Figure 6 for grayscale via transmission variable $y$ and Figure 7 for RGB via transmission variable $x$.
Also, it could be seen that the obtained results of the image encryption and transmission from both MATLAB and VHDL in Figures 6 and 7 are the same (see the encrypted and recovered image data). The time scale in the VHDL ModelSim results in Figures 6(b) and 7(b) is expressed in nanosecond (ns). The total simulation time was $700 \mathrm{~ns}$. A sample is generated every $0.002 \mathrm{~ns}$, making a total of $35 \times 10^{4}$ samples, which is equivalent to the MATLAB results expressed in number of samples in Figures 6(a) and 7(a).

In Figures 8 and 9 are found the actual original, encrypted, and recovered grayscale and RGB images obtained from the best results, respectively. The results of the chaotic image transmission system were examined with statistical analysis. The applied statistical tests are information entropy and correlation. The results are presented in Table 1.

As it is expected, a reliable encryption system should be robust enough to generate encrypted image with very high entropy and have correlation between original and encrypted image of zero. Therefore, in the statistical analysis results presented in Table 1, it is seen that the entropy is very high (very close to 8 ) in both grayscale image transmission and RGB image transmission. The higher the information entropy is, the more random the encrypted image is. This shows that the chaos-based encryption system is very effective. Also, the correlation between the original image and encrypted image in both cases is 0 . This shows that the encryption performed effectively removes relativity between the original and encrypted images. Moreover, the correlation between the original image and recovered image is 1 , meaning there is no loss of information. 

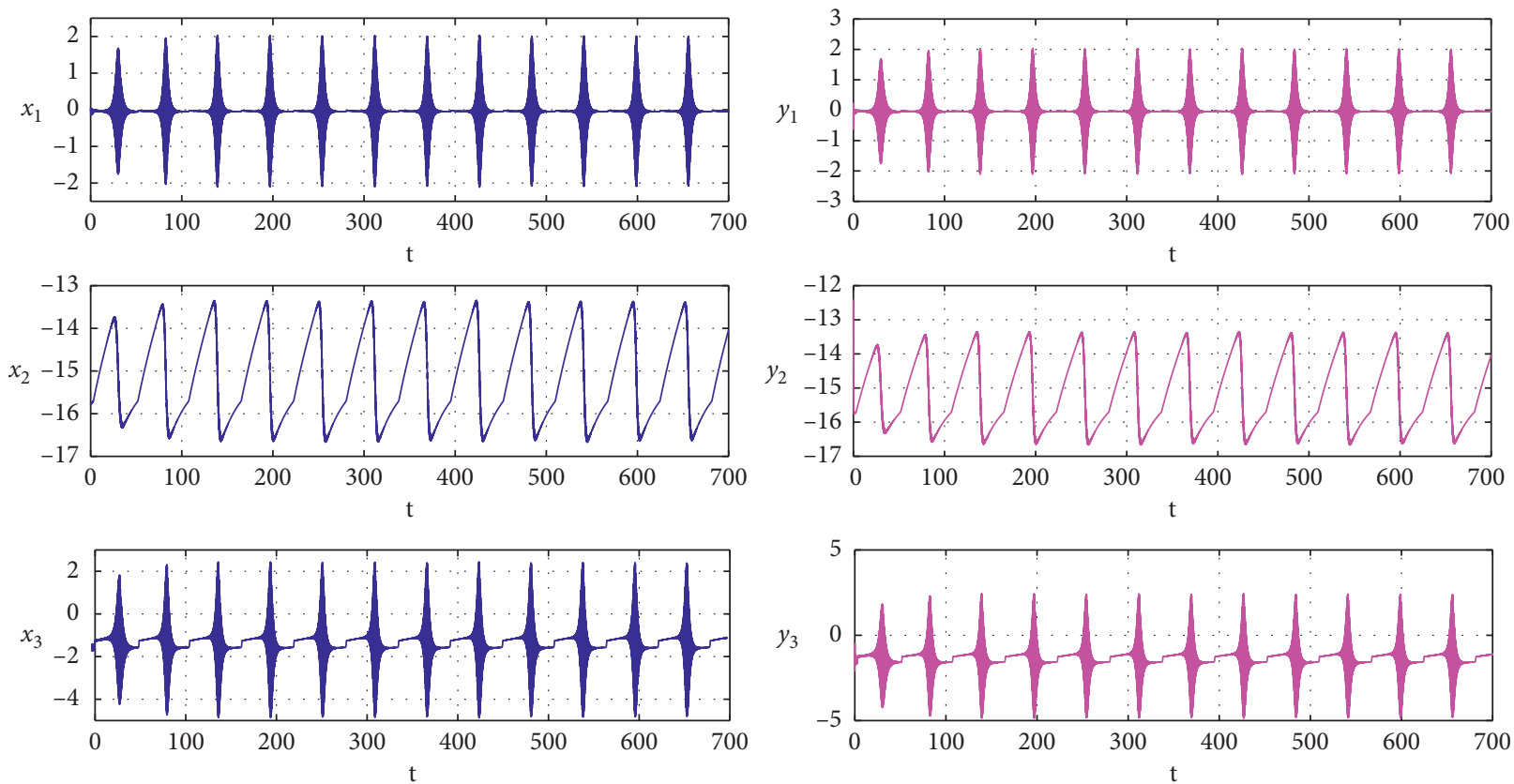

(a)

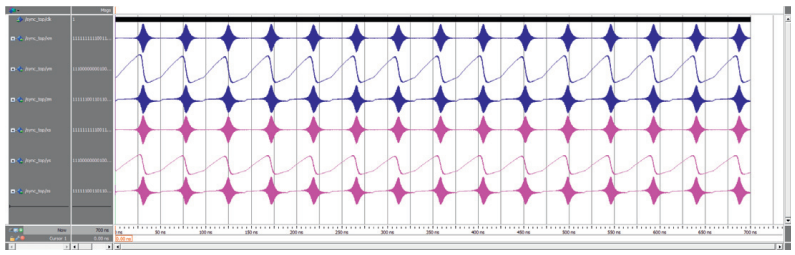

(b)

Figure 3: Waveforms of the master (blue) and slave (magenta) 3-D spherical chaotic systems. (a) MATLAB. (b) ModelSim.

4.3. FPGA Device Resources. In this investigation, the 3-D spherical attractor and the grayscale and RGB image transmissions are emulated on two FPGA boards, namely, Intel's Stratix III and Xilinx's Artix-7 AC701, using the same VHDL design codes. In the implementation of the image transmission, lookup tables (LUTs) are created for the input grayscale and RGB image vectors. The resources consumed on both boards in the full hardware synthesis are presented in this section.

In the case of Stratix III, the logic device design software used for the emulation of the activities on Intel's Stratix III board is the Quartus II 13.1 (64-bit) version, while the selected FPGA device in the Stratix III family is EP3SL340H1152C2. The device resources consumed by the FPGA emulation of the 3-D spherical attractor are given in Table 2, while for grayscale and RGB image transmissions, they are presented in Table 3.

On the other hand, for the Artix-7 AC701 board, Xilinx's Vivado version 2019.2 64-bit is used for the HDL design. Vivado device support is limited to 7th generation Xilinx FPGAs onwards, including Artix-7. The specific AC701 device used is XC7A200TFBG676-2. The resources consumed by the implementations of the 3-D spherical attractor as well as the grayscale and RGB image transmissions are presented in Tables 2 and 3, respectively.
Furthermore, in the FPGA emulation on Intel's Stratix III of the 3-D spherical oscillator and the grayscale and RGB image transmissions, the amount of dedicated logic registers utilized is less than one percent of the total available (see Tables 2 and 3). Also, the implementations do not use any memory LUTs and block RAMs. Also, it could be seen in Table 3 that the grayscale image transmission utilizes more logic resources than in RGB, as evidenced in the number of utilized LUTs. The overall logic utilization, which is an estimation of how full the device is, for grayscale image transmission is $85 \%$ while for the RGB, it is $79 \%$. Bearing in mind that both transmissions used the same design, the difference in logic utilization is attributed to the size of the images, which in the case of grayscale is made up of $640 \times 480=307,200$ words, compared to the RGB of $320 \times 240 * 3=230,400$ words.

Likewise, in the Xilinx's Artix-7 AC701 board, the logic registers used in the three implementations are less than one percent of the total available, no memory LUTs are used, and none of the 365 block RAMs ( $36 \mathrm{~kb}$ each) are utilized (see Tables 2 and 3). The utilization of the LUTs in Artix-7 AC701 for the image transmissions confirms also the result obtained in Stratix III (see Table 3) that the grayscale image transmission consumes more logic resources than in the transmission of RGB image because of the larger size of the grayscale image. 

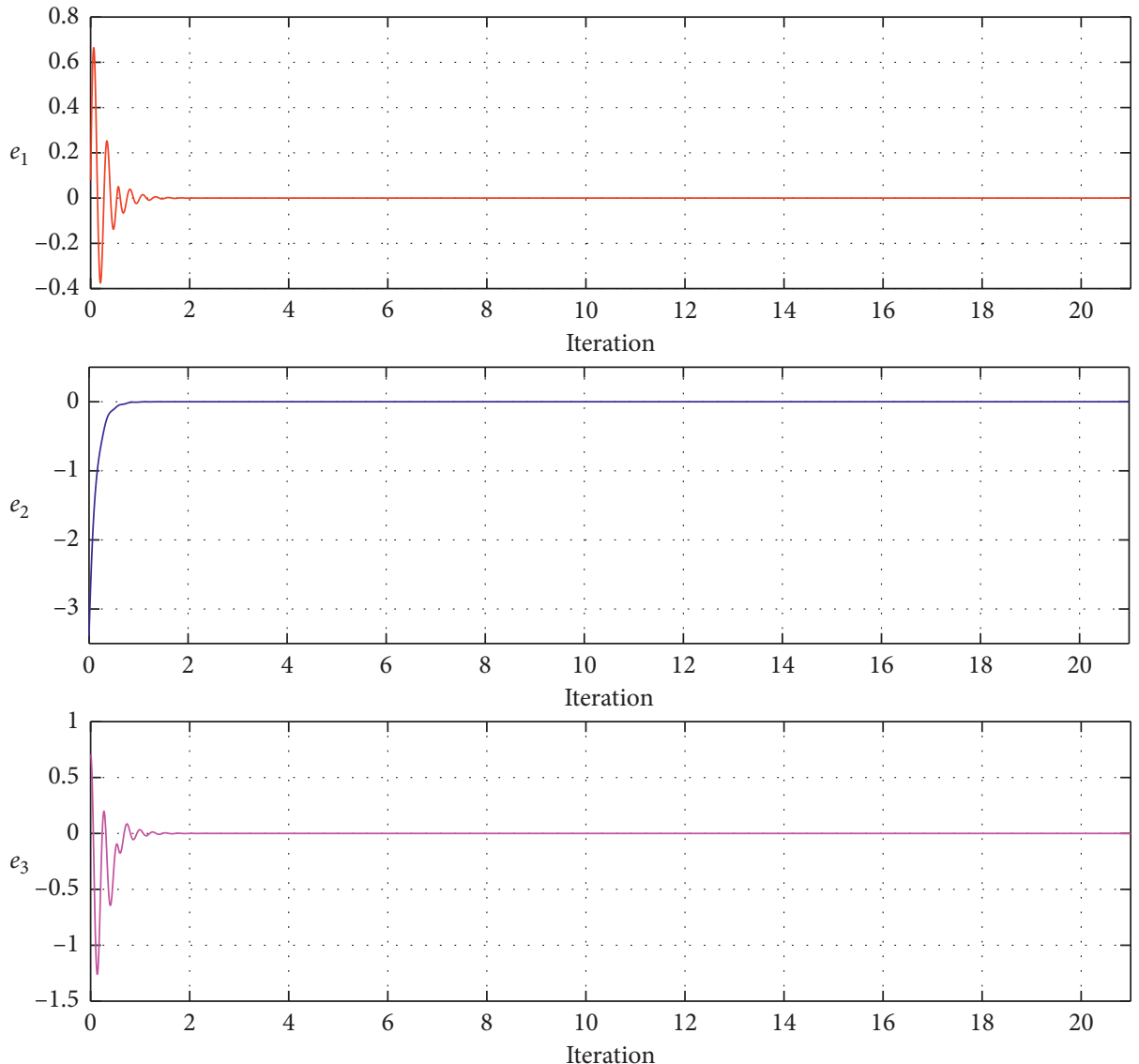

(a)

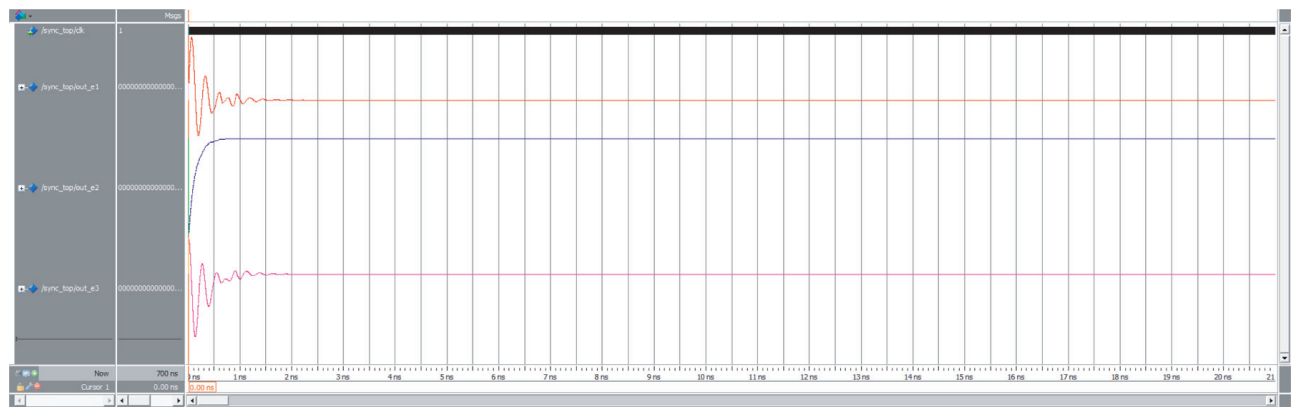

(b)

FIgURE 4: Synchronization errors of the master and slave systems. (a) MATLAB. (b) ModelSim.

\section{Discussion}

In comparing utilization of resources in the Xilinx Artix-7 AC701 with Intel's Stratix III, it is noted that the number of I/ O pins utilized for the implementations are the same: 97 for spherical attractor (see Table 2) and 99 each for the grayscale and RGB image transmissions (see Table 3). However, the Stratix III device consumes lesser LUTs and registers than Artix-7 AC701. The key to this better performance in Stratix III is its fracturable adaptive logic module (ALM), consisting of 8-input combinational logic, two registers, and two adders, which is more flexible and area-efficient than the logic unit of Artix-7 AC701, which consists of a basic 6-input LUT, carry chain, and two registers. On the other hand, the Artix-7
AC701 device has better performance in terms of the digital signal processing (DSP) block utilization. This is because Artix-7 AC701 DSP offers more efficient resource utilization and better speed and circuitry than the Stratix III device. The Artix-7 AC701 device supports clock rates of up to $628 \mathrm{MHz}$, up to 930 giga multiply-accumulate operations per second (GMACs), and up to 48-bit word lengths of data, compared to Stratix III's $550 \mathrm{MHz}, 537$ GMACs, and 36-bit word lengths, respectively. Overall, the results demonstrate that the 3-D spherical oscillator can be easily and efficiently implemented on FPGA technologies by providing real-time chaotic signals and attractors.

The FPGA implementation of the spherical chaotic oscillator on Stratix III (FPGA 1) and Artix-7 AC701 (FPGA 2) 


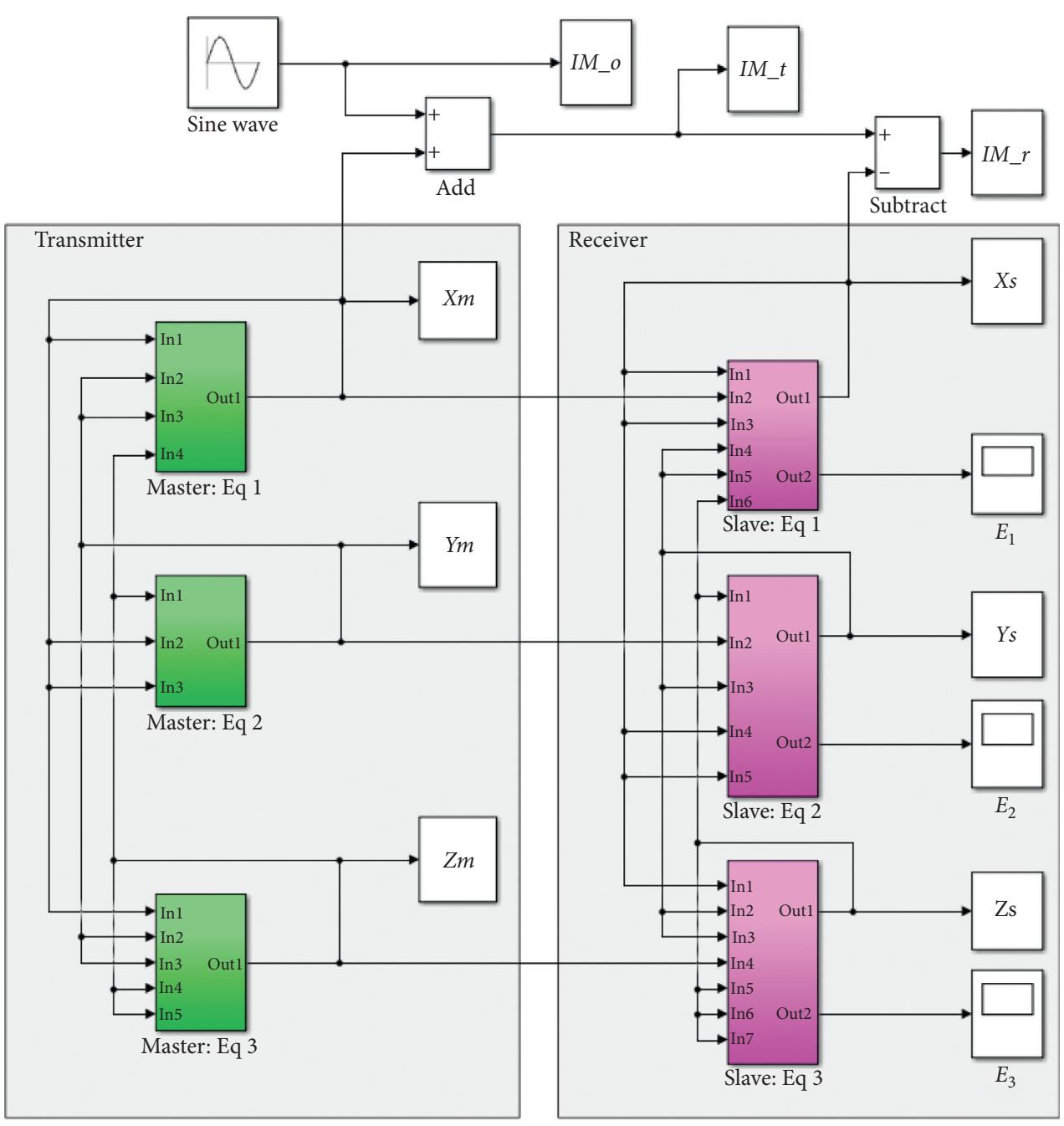

FIGURE 5: MATLAB-Simulink block diagram of the secure communication system implemented in master-slave topology.

TABLE 1: Statistical analysis of encrypted and recovered grayscale and RGB image transmissions.

\begin{tabular}{|c|c|c|c|}
\hline \multirow{2}{*}{ Transmission variable } & \multirow{2}{*}{ Entropy of encrypted image } & \multicolumn{2}{|c|}{ Correlation } \\
\hline & & Original and encrypted & Original and received \\
\hline \multicolumn{4}{|c|}{ Grayscale $640 \times 480$ pixels image } \\
\hline$X$ & 7.9977 & 0.0007 & 1 \\
\hline Y & 7.9977 & -0.0004 & 1 \\
\hline$Z$ & 7.9977 & 0.0030 & 1 \\
\hline \multicolumn{4}{|c|}{ RGB $320 \times 240$ pixels image } \\
\hline$X$ & 7.9988 & -0.0022 & 1 \\
\hline Y & 7.9989 & -0.0058 & 1 \\
\hline$Z$ & 7.9992 & 0.0026 & 1 \\
\hline
\end{tabular}

in this work is compared with some of the investigations that have been done relating to FPGA realization of chaotic oscillators [39, 58-60], using some of the resource parameters and other parameters, such as the FPGA board, applied numerical algorithm, implementation language, and the fixed-point number format. This is presented in Table 4 . The referenced works use the same 32-bit word length as in our implementations. It is noted that the model of the 3-D spherical chaotic oscillator used in this investigation is relatively more complex, with its hyperbolic tangent function and several nonlinear terms, than the referenced works. However, references [39, 58, 60], which implement less complex Lü-Chen, hyperchaotic Lorenz, and rotated Lorenz chaotic models, utilize more registers $(2 \%, 5 \%$, and $49 \%$, respectively) and more I/O pins (41\%, 32\%, and 36\%, respectively) than both realizations in this work. Also, references [57, 59] consumed more LUTs (24\% and 101\%, respectively) than our realizations on both Stratix III and Artix-7 AC701, while the utilization of the DSPs in reference [57] is higher than that of our implementation on Artix-7 

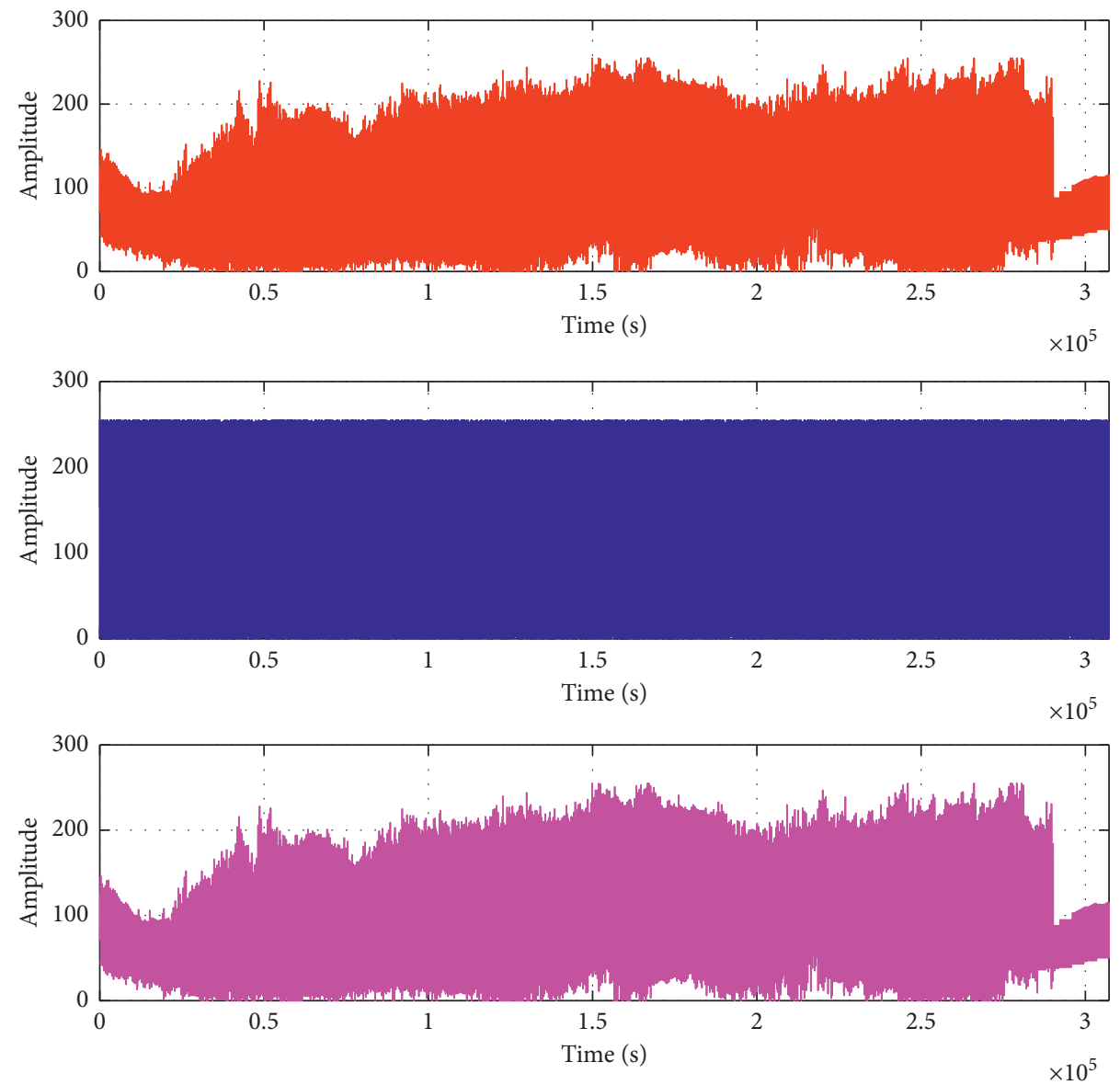

(a)

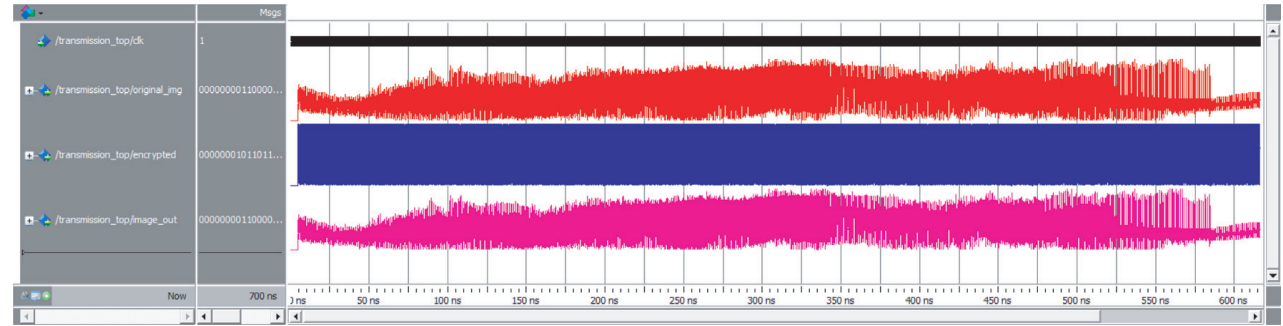

(b)

Figure 6: Waveforms of $640 \times 480$ pixels grayscale image transmission via state variable $y$. (a) MATLAB. (b) ModelSim.

AC701 and almost the same as Stratix III. Reference [58] uses the same LUTs and registers [ $3 \%$ and $<1 \%$, respectively] as in our Stratix III realization. Overall, the FPGA resource consumption on Stratix III and Artix-7 AC701 in this work compares favorably with most of the listed references despite having a more complex structure.

The chaos-based secure communication system implemented in this work has some basic advantages over conventional techniques, such as spread spectrum, least significant bit (LSB), and echo methods. For example, one of the advantages is its simplicity in that the system is implemented by using only one subsystem at each end of the communication link, which are the master and slave subsystems. Despite this simplicity, the chaos-based secure communication system provides all the basic processes required for digital message transmission. On the other hand, the conventional systems require enormous use of various subsystems. Also, the technique involved in the chaos-based communication system does not require different tools for synchronization and the image encryption, as the chaotic states of the oscillator are used for both the synchronization and the encryption. Moreover, the chaotic signals have impulsive-like autocorrelations and low crosscorrelation values. Another edge that the chaos-based secure communication system has is that it is more robust against noises and distortions [61].

Furthermore, the chaos-based image encryption and decryption scheme in this work is faster because it requires only one round to perform each of the operations, unlike the well-known encryption algorithms such as the AES, DES, 

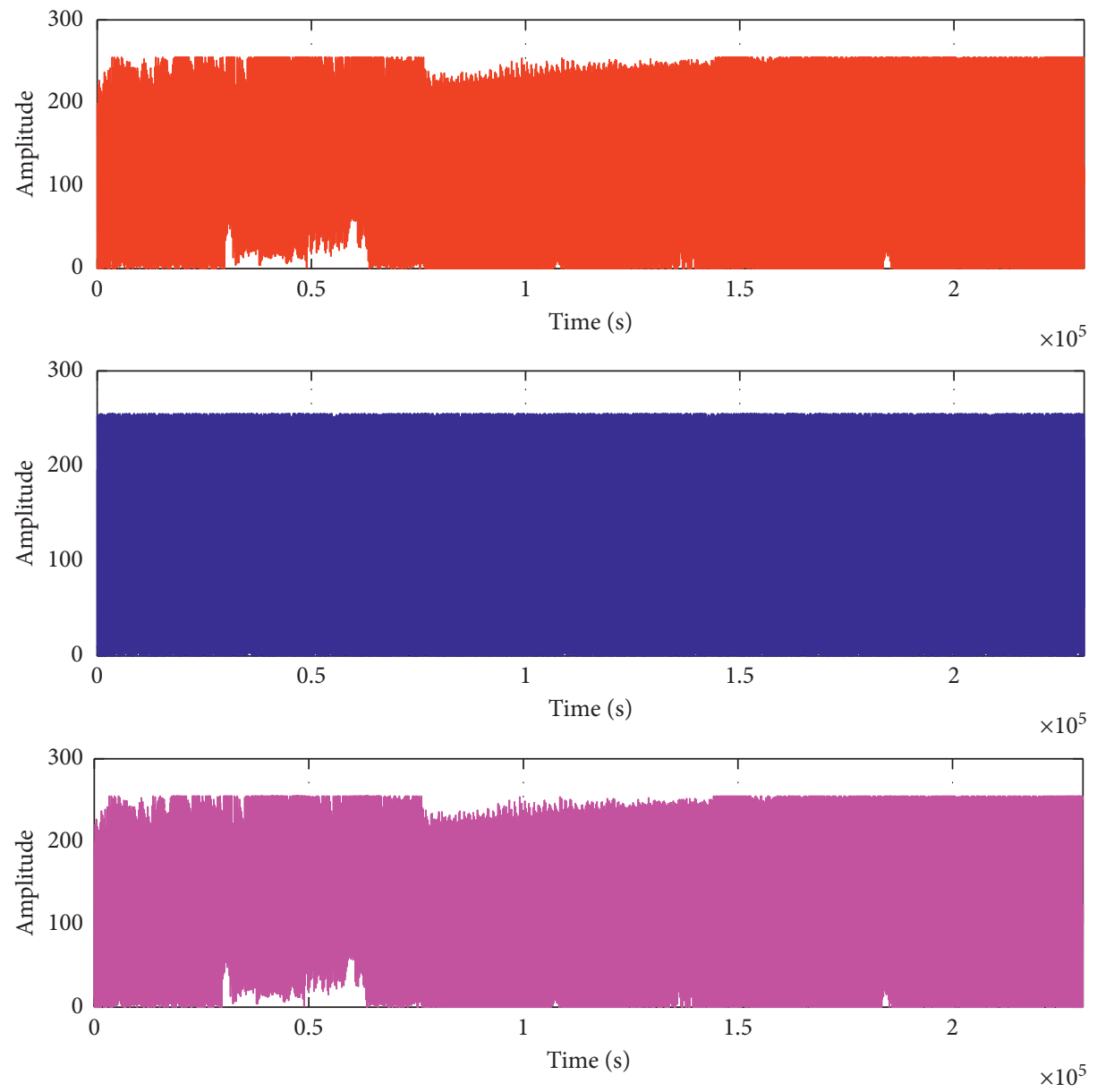

(a)

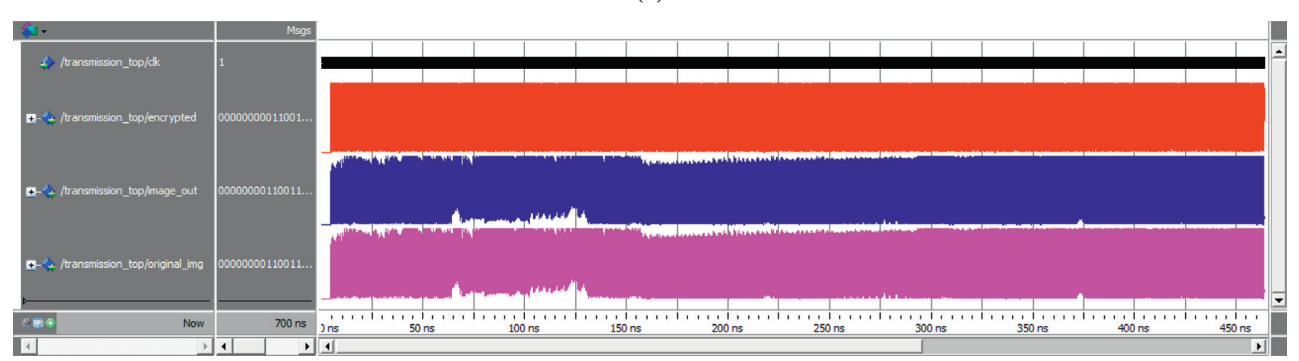

(b)

Figure 7: Waveforms of $320 \times 240$ pixels RGB image transmission via state variable $x$. (a) MATLAB. (b) ModelSim.

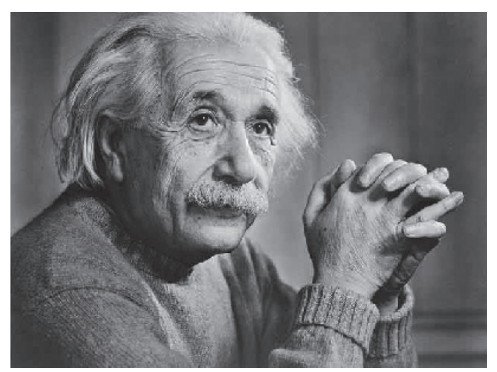

(a)

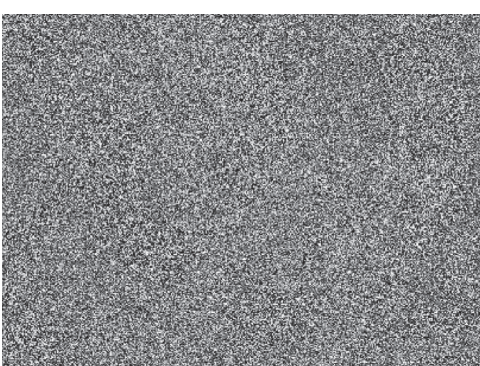

(b)

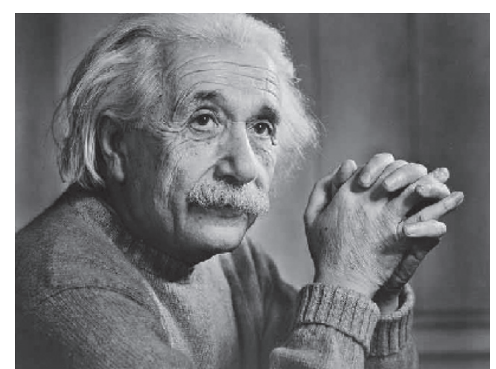

(c)

FiguRe 8: Grayscale image transmission result via state variable $y$ : original (a), encrypted (b), and recovered (c). 


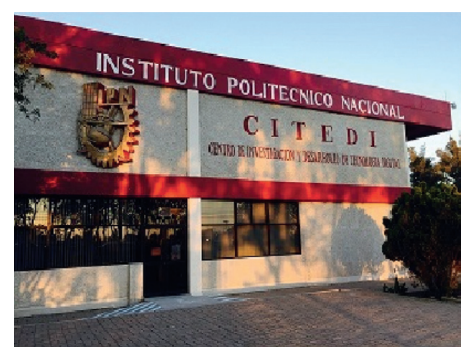

(a)

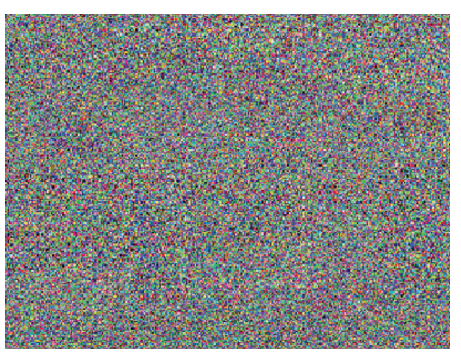

(b)

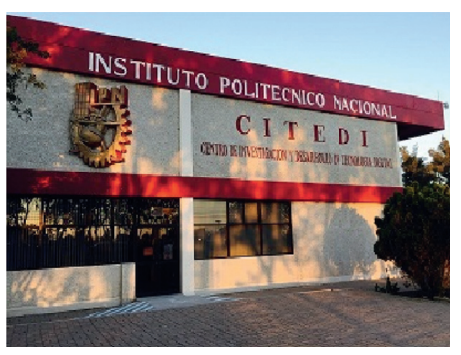

(c)

FIgURE 9: RGB image transmission result via state variable $x$ : original (a), encrypted (b), and recovered (c).

TABLE 2: Utilization of resources in spherical attractor implementation on Intel's Stratix III and Xilinx's Artix-7 AC701 FPGA boards.

\begin{tabular}{|c|c|c|c|c|c|c|}
\hline \multirow{2}{*}{ Resources } & \multicolumn{3}{|c|}{ FPGA 1: Intel Stratix III } & \multicolumn{3}{|c|}{ FPGA 2: Xilinx Artix-7 AC701 } \\
\hline & Available & Used & Utilization (\%) & Available & Used & Utilization (\%) \\
\hline LUTs & 270,400 & 10,261 & 4 & 215,360 & 13,228 & 10 \\
\hline Memory LUTs & 135,200 & 0 & 0 & 46,200 & 0 & 0 \\
\hline Registers & 270,400 & 192 & $<1$ & 269,200 & 193 & $<1$ \\
\hline $\mathrm{I} / \mathrm{O}$ pins & 744 & 97 & 13 & 400 & 97 & 24 \\
\hline Block RAMs & $16,662,528$ & 0 & 0 & $13,140,000$ & 0 & 0 \\
\hline DSPs & 576 & 368 & 64 & 208 & 208 & 28 \\
\hline
\end{tabular}

TABLE 3: Utilization of resources in grayscale and RGB image transmissions on Intel's Stratix III and Xilinx's Artix-7 AC701 FPGA boards.

\begin{tabular}{|c|c|c|c|c|c|}
\hline \multirow{3}{*}{ Resources } & \multicolumn{5}{|c|}{ FPGA 1: Intel Stratix III } \\
\hline & \multirow{2}{*}{ Available } & \multicolumn{2}{|c|}{ Grayscale } & \multicolumn{2}{|c|}{ RGB } \\
\hline & & Used & Utilization (\%) & Used & Utilization (\%) \\
\hline LUTs & 270,400 & 133,939 & 50 & 125,144 & 46 \\
\hline Memory LUTs & 135,200 & 0 & 0 & 0 & 0 \\
\hline Registers & 270,400 & 607 & $<1$ & 607 & $<1$ \\
\hline $\mathrm{I} / \mathrm{O}$ pins & 744 & 99 & 13 & 99 & 13 \\
\hline Block RAMs & $16,662,528$ & 0 & 0 & 0 & 0 \\
\hline DSPs & 576 & 576 & 100 & 576 & 100 \\
\hline \multicolumn{6}{|c|}{ FPGA 2: Xilinx Artix-7 AC701 } \\
\hline LUTs & 215,360 & 150,830 & 70 & 143,183 & 66 \\
\hline Memory LUTs & 46,200 & 0 & 0 & 0 & 0 \\
\hline Registers & 269,200 & 1,333 & $<1$ & 1,334 & $<1$ \\
\hline $\mathrm{I} / \mathrm{O}$ pins & 400 & 99 & 25 & 99 & 25 \\
\hline Block RAMs & $13,140,000$ & 0 & 0 & 0 & 0 \\
\hline DSPs & 208 & 424 & 57 & 424 & 57 \\
\hline
\end{tabular}

TABle 4: Comparison of the spherical oscillator implementations on Stratix III and Artix-7 AC701 with other implementations.

\begin{tabular}{|c|c|c|c|c|c|c|}
\hline Parameter & \multicolumn{2}{|c|}{ This work } & Ref. [39] & Ref. [58] & Ref. [59] & Ref. [60] \\
\hline FPGA & Stratix III & Artix-7 & Virtex 6 & Virtex 5 & Cyclone 4 & Virtex 6 \\
\hline LUTs & $4 \%$ & $10 \%$ & $4 \%$ & $24 \%$ & $3 \%$ & $101 \%$ \\
\hline Registers & $<1 \%$ & $<1 \%$ & $2 \%$ & $5 \%$ & $<1 \%$ & $49 \%$ \\
\hline $\mathrm{I} / \mathrm{O}$ pins & $13 \%$ & $24 \%$ & $41 \%$ & $32 \%$ & $8 \%$ & $36 \%$ \\
\hline DSPs & $64 \%$ & $28 \%$ & $22 \%$ & $62 \%$ & $9 \%$ & N/A \\
\hline Algorithm & RK-4 & RK-4 & Heun & RK-4 & N/A & RK-4 \\
\hline Language & VHDL & VHDL & VHDL & VHDL & Verilog & VHDL \\
\hline Number & 32-bit & 32-bit & 32-bit & 32-bit & 32-bit & 32-bit \\
\hline
\end{tabular}

and IDEA, which have low-level efficiency in encryption and decryption process [62]. The AES, for example, requires 10 rounds of encryption. Also, the chaos-based encryption algorithm has a large key space because of the sensitivity of the chaotic system to the initial values and the parameters, which makes the algorithm more robust against security attacks. In addition, the chaotic states are pseudorandom, and hence the encryption algorithm provides a more randomized image by removing relativity with the original image. 


\section{Conclusions}

This work was done to further demonstrate the suitability of FPGA for the electronic implementation of a chaotic oscillator. In a first major contribution, it was shown the FPGA realization of a 3-D system generating spherical chaotic attractors. Furthermore, two spherical chaotic systems were synchronized in a master-slave topology and emulated on a FPGA device. The synchronization was successfully achieved by Hamiltonian forms and observer approach. The master and the slave systems synchronized very fast during which the synchronization error becomes zero. Lastly, it was demonstrated also by FPGA emulation the transmission of grayscale and RGB images using the synchronized master and slave systems. The design process of the FPGA was performed using VHDL on Intel's Stratix III and Xilinx's Artix-7 AC701 boards. The VHDL-based results obtained via ModelSim are the same as the MATLAB results. The statistical analyses of the image transmission results show that there is no relativity between the original and encrypted grayscale and RGB images, as the correlation coefficient is 0 in all the transmission variables. Moreover, the correlation between the adjacent pixels examined in horizontal, vertical, and diagonal directions in the encrypted grayscale and RGB images is 0 . The original images were completely recovered without loss of information, since the correlation coefficient between the original and recovered grayscale and RGB images is 1 . Hence, the implemented transmission system is a secure communication system.

\section{Data Availability}

The data used to support the findings of this study are available from the corresponding author upon request.

\section{Conflicts of Interest}

The authors declare that there are no conflicts of interest regarding the publication of this paper.

\section{Acknowledgments}

The authors wish to thank Instituto Politécnico Nacional for its support provided through the project "SIP-20210345."

\section{References}

[1] A. Belmiloudi, "Dynamical behavior of nonlinear impulsive abstract partial differential equations on networks with multiple time-varying delays and mixed boundary conditions involving time-varying delays," Journal of Dynamical and Control Systems, vol. 21, no. 1, pp. 95-146, 2015.

[2] M. V. Demina and N. S. Kuznetsov, "Liouvillian integrability and the Poincaré problem for nonlinear oscillators with quadratic damping and polynomial forces," Journal of Dynamical and Control Systems, vol. 27, pp. 403-415, 2021.

[3] A. L. Dawidowicz and A. Poskrobko, "On chaos behaviour of nonlinear Lasota equation in Lebesgue space," Journal of Dynamical and Control Systems, vol. 27, pp. 371-378, 2021.

[4] G. Nicolis, "Chapter 1 -the many facets of complexity," in Complexity Science: An Introduction, M. A. Peletier,
R. A. van Santen, and E. Steur, Eds., World Scientific Publishing Co., pp. 3-30, Singapore, 2019, ISBN: 978-981323960-9.

[5] J. Iqbal, M. Ullah, S. Khan, B. Khelifa, and S. Cuković, "Nonlinear control systems - a brief overview of historical and recent advances," Nonlinear Engineering, vol. 6, no. 4, pp. 301-312, 2017.

[6] P. Mellodge, "Chapter 4 - characteristics of nonlinear systems," in A Practical Approach to Dynamical Systems for Engineers, P. Mellodge, Ed., Woodhead Publishing, pp. 215-250, Sawston, England, 2016, ISBN 9780081002025.

[7] W. K. Ling, Nonlinear Digital Filters: Analysis and Applications, Academic Press, Cambridge, MA, USA, 2010, ISBN 9780080550015.

[8] L. Moysis, M. K. Gupta, V. Mishra, M. Marwan, and C. Volos, "Observer design for rectangular descriptor systems with incremental quadratic constraints and nonlinear outputs: application to secure communications," International Journal of Robust and Nonlinear Control, vol. 30, no. 18, pp. 81398158, 2020.

[9] Y. Tian and Z. Wang, "Stability analysis and generalised memory controller design for delayed T-S fuzzy systems via flexible polynomial-based functions," IEEE Transactions on Fuzzy Systems (Early Access), p. 1, 2020.

[10] Y. Tian and Z. Wang, "Finite-time extended dissipative filtering for singular T-S fuzzy systems with nonhomogeneous markov jumps," IEEE Transactions on Cybernetics (Early Access), pp. 1-11, 2020.

[11] Y. Tian and Z. Wang, "Extended dissipativity analysis for markovian jump neural networks via double-integral-based delay-product-type Lyapunov functional," IEEE Transactions on Nueral Networks and Learning Systems (Early Access), pp. 1-7, 2020.

[12] D. Chowdhury and H. K. Khalil, "Practical synchronization in networks of nonlinear heterogeneous agents with application to power systems," IEEE Transactions on Automatic Control, vol. 66, no. 1, pp. 184-198, 2021.

[13] S. Ozcan, M. U. Salamci, and V. Nalbantoglu, "Nonlinear sliding sector design for multi-input systems with application to helicopter control," International Journal of Robust and Nonlinear Control, vol. 30, no. 6, pp. 2248-2291, 2020.

[14] T. Zhang, F. Kratz, Y. Hou, and V. Idasiak, "A continuousdiscrete finite memory observer design for a class of nonlinear systems: application to fault diagnosis," Mathematical Problems in Engineering, vol. 2020, Article ID 7312521, 14 pages, 2020.

[15] H. Min, S. Xu, S. Fei, G. Cui, and Y. Tan, "Observer-based tracking control for constrained nonlinear systems with mismatching disturbances and its application," International Journal of Robust and Nonlinear Control, vol. 30, no. 18, pp. 8485-8502, 2020.

[16] X. Yao and Y. Yang, "Adaptive fault compensation and disturbance suppression design for nonlinear systems with an aircraft control application," International Journal of Aerospace Engineering, vol. 2020, Article ID 4531302, 16 pages, 2020.

[17] W. Wang, R. Postoyan, D. Nešić, and W. P. M. H. Heemels, "Periodic event-triggered control for nonlinear networked control systems," IEEE Transactions on Automatic Control, vol. 65 , no. 2, pp. 620-635, 2020.

[18] L. Dai and R. N. Jazar, Nonlinear Approaches in Engineering Applications, Springer, Berlin, Germany, 2012, ISBN 978-14614-1468-1.

[19] E. N. Lorenz, "Deterministic nonperiodic flow," Journal of the Atmospheric Sciences, vol. 20, no. 2, pp. 130-141, 1963. 
[20] G. Chen, "The Chen system revisited," Dynamics of Continuous, Discrete and Impulsive Systems, Series B: Applications \& Algorithms, vol. 20, pp. 691-696, 2013.

[21] O. E. Rössler, "Different types of chaos in two simple differential equations," Zeitschrift Fur Naturforschung A, vol. 31, no. 12, pp. 1976-1231, 1976.

[22] O. E. Rössler, "Continuous chaos-four prototype equations," Annals of the New York Academy of Sciences, vol. 316, no. 1, pp. 376-392, 1979.

[23] J. C. Sprott, "Some simple chaotic flows," Physical Review E, vol. 50, no. 2, pp. R647-R650, 1994.

[24] G. Chen and X. Yu, Chaos Control: Theory and Applications, Springer Science \& Business Media, Berlin, Germany, 2003.

[25] G. Li and X. Chen, "Constructing piecewise linear chaotic system based on the heteroclinic Shil'nikov theorem," Communications in Nonlinear Science and Numerical Simulation, vol. 14, no. 1, pp. 194-203, 2009.

[26] S. Grandner, S. Heidenreich, S. Hess, and S. H. L. Klapp, "Polar nano-rods under shear: from equilibrium to chaos," The European Physical Journal E, vol. 24, no. 4, pp. 353-365, 2007.

[27] T. Khapko, Y. Duguet, T. Kreilos, P. Schlatter, B. Eckhardt, and D. S. Henningson, "Complexity of localised coherent structures in a boundary-layer Flow," European Physical Journal E, vol. 37, no. 4, p. 32, 2014.

[28] M. Eisencraft, R. Attux, and R. Suyama, Chaotic Signals in Digital Communications, CRC Press, Boca Raton, FL, USA, 2018.

[29] T.-L. Liao, C.-Y. Chen, H.-C. Chen, Y.-Y. Chen, and Y.-Y. Hou, "Realization of a secure visible light communication system via chaos synchronization," Mathematical Problems in Engineering, vol. 2021, Article ID 6661550, 12 pages, 2021.

[30] L. Huang, D. Shi, and J. Gao, "The design and its application in secure communication and image encryption of a new lorenzlike system with varying parameter," Mathematical Problems in Engineering, vol. 2016, Article ID 8973583, 11 pages, 2016.

[31] S. Pang, Y. Feng, and Y. Liu, "Finite-time synchronization of chaotic systems with different dimension and secure communication," Mathematical Problems in Engineering, vol. 2016, Article ID 7693547, 14 pages, 2016.

[32] K. M. Cuomo and A. V. Oppenheim, "Circuit implementation of synchronized chaos with applications to communications," Physical Review Letters, vol. 71, no. 1, pp. 65-68, 1993.

[33] J. Lü and G. Chen, "Generating multiscroll chaotic attractors: theories, methods and applications," International Journal of Bifurcation and Chaos, vol. 16, no. 4, pp. 775-858, 2006.

[34] J. Lu, S. Yu, H. Leung, and G. Chen, "Experimental verification of multidirectional multiscroll chaotic attractors," IEEE Transactions on Circuits and Systems I: Regular Papers, vol. 53, no. 1, pp. 149-165, 2006.

[35] T. Gao, G. Chen, Z. Chen, and S. Cang, "The generation and circuit implementation of a new hyper-chaos based upon Lorenz system," Physics Letters A, vol. 361, no. 1-2, pp. 78-86, 2007.

[36] G. Qi and G. Chen, "Analysis and circuit implementation of a new 4D chaotic system,” Physics Letters A, vol. 352, no. 4-5, pp. 386-397, 2006.

[37] J. M. Muñoz-Pacheco, E. Tlelo-Cuautle, I. Toxqui-Toxqui, C. Sánchez-López, and R. Trejo-Guerra, "Frequency limitations in generating multi-scroll chaotic attractors using CFOAs," International Journal of Electronics, vol. 101, no. 11, pp. 1559-1569, 2014.

[38] E. Tlelo-Cuautle, A. J. Quintas-Valles, L. G. de la Fraga, and J. J. Rangel-Magdaleno, "VHDL descriptions for the FPGA implementation of PWL-function-based multiscroll chaotic oscillators," PLoS One, vol. 11, no. 2, p. e0168300, 2019.

[39] M. Tuna, M. Alçın, İ. Koyuncu, C. B. Fidan, and İ. Pehlivan, "High speed FPGA-based chaotic oscillator design," Microprocessors and Microsystems, vol. 66, pp. 72-80, 2019.

[40] Y. Cheng-Hsiung and H. Sih-Jie, "Secure color image encryption algorithm based on chaotic signals and its FPGA realization," International Journal of Circuit Theory and Applications, vol. 46, no. 12, pp. 2444-2461, 2018.

[41] M. Alcin, I. Koyuncu, M. Tuna, M. Varan, and I. Pehlivan, “A novel high speed artificial neural network-based chaotic true random number generator on field programmable gate array," International Journal of Circuit Theory and Applications, vol. 47, no. 3, pp. 365-378, 2019.

[42] E. Tlelo-Cuautle, L. G. De la Fraga, V.-T. Pham, C. Volos, S. Jafari, and A. D. J. Quintas-Valles, "Dynamics, FPGA realization and application of a chaotic system with an infinite number of equilibrium points," Nonlinear Dynamics, vol. 89, no. 2, pp. 1129-1139, 2017.

[43] P. Li, W. Zhang, Z. Li, W. Liu, and W. A. Halang, "FPGA implementation of a coupled-map-lattice-based cryptosystem," International Journal of Circuit Theory and Applications, vol. 38, no. 1, pp. 85-98, 2010.

[44] F. Yu, L. Liu, B. He et al., "Analysis and FPGA realization of a novel 5D hyperchaotic four-wing memristive system, active control synchronization, and secure communication application," Complexity, vol. 2019, Article ID 4047957, 18 pages, 2019.

[45] M. S. Azzaz, C. Tanougast, A. Maali, and M. Benssalah, "An efficient and lightweight multi-scroll chaos-based hardware solution for protecting fingerprint biometric templates," International Journal of Communication Systems, vol. 33, no. 10, p. e4211, 2020.

[46] E. A. A. Hagras and M. Saber, "Low power and high-speed FPGA implementation for 4D memristor chaotic system for image encryption," Multimedia Tools and Applications, vol. 79, no. 8, pp. 23203-23222, 2020.

[47] O. Guillén-Fernández, A. Meléndez-Cano, E. Tlelo-Cuautle, J. C. Núñez-Pérez, and J. J. Rangel-Magdaleno, "On the synchronization techniques of chaotic oscillators and their FPGA-based implementation for secure image transmission," PLoS One, vol. 14, no. 2, p. e0209618, 2019.

[48] E. Tlelo-Cuautle, J. D. Díaz-Muñoz, A. M. González-Zapata et al., "Chaotic image encryption using hopfield and hindmarsh-rose neurons implemented on FPGA," Sensors, vol. 20, no. 5, p. 1326, 2020.

[49] E. Tlelo-Cuautle, A. D. Pano-Azucena, O. Guillén-Fernández, and A. Silva-Juárez, "Synchronization and applications of fractional-order chaotic systems," Analog/Digital Implementation of Fractional Order Chaotic Circuits and Applications, Springer, Cham, Switzerland, 2019.

[50] R. Sivaraman, S. Rajagopalan, and R. Amirtharajan, "FPGA based generic RO TRNG architecture for image confusion," Multimedia Tools and Applications, vol. 79, no. 2, pp. 13841-13868, 2020.

[51] Z. Wang, Y. Sun, and S. Cang, "A 3-D spherical chaotic attractor," Acta Physica Polonica B, vol. 42, no. 2, pp. 235-247, 2011.

[52] J. C. Núñez Pérez, V. A. Adeyemi, S. E. Gutierrez Osuna, Y. Sandoval Ibarra, and E. Tlelo Cuautle, "Secure communication system based on synchronized 3D spherical chaotic systems," in Proceedings of the IEEE International Conference on Engineering Veracruz (ICEV), pp. 26-29, Boca del Rio, Mexico, October 2020. 
[53] T. Zhou and G. Chen, "A simple smooth chaotic system with a 3-layer attractor," International Journal of Bifurcation and Chaos, vol. 14, no. 5, pp. 1795-1799, 2004.

[54] L. P. Shilnikov, "A case of the existence of a countable number of periodic motions," Soviet Mathematics - Doklady, vol. 6, pp. 163-166, 1965.

[55] L. P. Shilnikov, "A contribution to the problem of the structure of an extended neighbourhood of a rough equilibrium state of saddle-focus type," Mathematics of the USSRSbornik, vol. 10, no. 1, pp. 91-102, 1970.

[56] H. Sira-ramírez and C. Cruz-hernández, "Synchronization of chaotic systems: a generalized Hamiltonian systems approach," International Journal of Bifurcation and Chaos, vol. 11, no. 5, pp. 1381-1395, 2001.

[57] L. J. Pei and S. H. Liu, "Application of generalized Hamiltonian systems to chaotic synchronization," Nonlinear Dynamics and Systems Theory, vol. 9, no. 4, pp. 415-432, 2009.

[58] S. Sadoudi, C. Tanougast, M. S. Azzaz, and A. Dandache, "Design and FPGA implementation of a wireless hyperchaotic communication system for secure real-time image transmission," EURASIP Journal on Image and Video Processing, vol. 2013, p. 43, 2013.

[59] W. S. Sayed, A. G. Radwan, M. Elnawawy et al., "Two-dimensional rotation of chaotic attractors: demonstrative examples and FPGA realization," Circuits, Systems, and Signal Processing, vol. 38, no. 10, pp. 4890-4903, 2019.

[60] M. Tuna and C. B. Fidan, "Electronic circuit design, implementation and FPGA-based realization of a new 3D chaotic system with single equilibrium point," Optik, vol. 127 , no. 24 , pp. 11786-11799, 2016.

[61] V. G. Gribunin, I. N. Okov, and I. V. Turincev, Cifrovaya Steganografiya. [Digital Steganography], p. 262, M: SOLONPress, Berlin, Germany, 2017.

[62] S. Koppu, V. M. Viswanatham, A fast enhanced secure image chaotic cryptosystem based on hybrid chaotic magic transform," Modelling and Simulation in Engineering, vol. 2017, Article ID 7470204, 12 pages, 2017. 